\title{
Norois
}

Environnement, aménagement, société

$195 \mid 2005 / 2$

L'appropriation de l'espace : sur la dimension spatiale des inégalités sociales et des rapports de pouvoir

\section{Le recyclage urbain, entre démolition et patrimonialisation : enjeux d'appropriation symbolique de l'espace}

Réflexions à partir de quatre villes de l'Ouest

Urban recycling between destruction and conservation: the symbolic

appropriation stakes of space. Reflections based on the examples of four towns of the West of France

\section{Vincent Veschambre}

\section{OpenEdition}

Journals

\section{Édition électronique}

URL : https://journals.openedition.org/norois/548

DOI : $10.4000 /$ norois. 548

ISBN : 978-2-7535-1543-7

ISSN : 1760-8546

Éditeur

Presses universitaires de Rennes

Édition imprimée

Date de publication : 1 juin 2005

Pagination : 79-92

ISBN : $978-2-7535-0123-2$

ISSN : 0029-182X

Référence électronique

Vincent Veschambre, «Le recyclage urbain, entre démolition et patrimonialisation : enjeux

d'appropriation symbolique de l'espace », Norois [En ligne], 195 | 2005/2, mis en ligne le 08 août 2008, consulté le 21 septembre 2021. URL : http://journals.openedition.org/norois/548 ; DOI : https:// doi.org/10.4000/norois.548

Ce document a été généré automatiquement le 21 septembre 2021.

(c) Tous droits réservés 


\section{Le recyclage urbain, entre démolition et patrimonialisation : enjeux d'appropriation symbolique de l'espace}

Réflexions à partir de quatre villes de l'Ouest

Urban recycling between destruction and conservation: the symbolic

appropriation stakes of space. Reflections based on the examples of four towns of the West of France

Vincent Veschambre

\section{NOTE DE L'ÉDITEUR}

Cet article a été reçu le 20 décembre 2004 et définitivement accepté le 30 mai 2005.

1 La question de l'appropriation de l'espace est révélatrice des relations de pouvoir entre groupes sociaux. Nous l'envisagerons dans le cadre de la ville, «création du pouvoir et des lieux de pouvoir» (Burgel, 2001), espace où ces enjeux d'appropriation sont exacerbés. Manifestation du principe de centralité, la ville est par excellence le théâtre de conflits pour l'appropriation d'espaces rares.

2 Sans pour autant l'occulter dans notre réflexion, nous n'envisagerons pas cette question de l'appropriation de l'espace urbain sous l'angle juridique des transferts de propriété, ou sous l'angle économique de la valeur et de ses évolutions. Mais de manière privilégiée sous l'angle symbolique de la légitimité des acteurs sociaux en présence et des revendications de visibilité qu'ils portent dans cet espace de mise en scène des pouvoirs. Même si nous avons conscience, comme l'écrivait J.-P. Lévy (1987) à propos du centre ville, que « les valeurs culturelles, immatérielles, se combinent étroitement aux valeurs matérielles» et que "fortement chargé de valeurs et de significations, avivées et constamment renouvelées par les rivalités pour son appropriation, l'espace central est à 
la fois un espace symbole et un espace enjeu ", nous avons tenu à privilégier la lecture symbolique des transformations urbaines, afin de compléter des approches plus classiques en géographie sociale, prioritairement axées sur les mécanismes fonciers et plus largement économiques (Dynamiques urbaines, 1991). Et cela sans perdre de vue les inégalités sociales et les rapports de domination qui sont l'objet même de cette manière de faire la géographie.

3 Nous avons eu l'occasion d'affirmer cette hypothèse selon laquelle la dimension symbolique était inhérente à toutes formes d'appropriation, y compris les plus violentes (Veschambre, 2004). Par son inscription dans la durée, la production architecturale est par excellence le mode d'affirmation symbolique du pouvoir sur un espace: « les classes dominantes inscrivent dans la pierre et par l'architecture leur réussite économique et leur domination sociale et politique " (Chevalier et al., 1984). C'est donc dans ce registre $\mathrm{du}$ symbolique que nous envisagerons les interventions sur le tissu urbain et les formes d'appropriation qui sont alors exprimées. Nous souhaitons contribuer à mieux cerner en quoi la démolition et son envers, la conservation (monumentalisation, patrimonialisation pour ce qui relève de la période la plus récente) constituent les deux faces d'un même processus de recyclage et de réappropriation à la fois matérielle et symbolique de l'espace urbain, notamment dans ces moments stratégiques que constituent les changements d'usage d'un édifice ou d'un ensemble urbain.

4 Cette réflexion sera nourrie par des exemples travaillés dans le cadre d'une comparaison des politiques patrimoniales des villes de l'Ouest: nous évoquerons principalement les terrains d'Angers, du Mans, de Nantes et de Caen ${ }^{1}$. Afin d'alimenter la réflexion sur cette dialectique patrimonialisation/démolition, nous insisterons sur le traitement réservé aux héritages les plus récents et les moins reconnus, les héritages industriels notamment, traitement qui s'avère différencié d'une ville à l'autre.

\section{La ville : un balancement permanent entre destruction et conservation}

\section{La ville : une éternelle reconstruction}

5 L'expression de "renouvellement urbain", traduite en langage courant par "reconstruire la ville sur la ville », s'est imposée à la fin $d u x^{e}$ siècle, pour désigner les formes de recyclage des espaces urbains désaffectés ou jugés obsolètes, dans les villes des pays anciennement industrialisés (Bergel, 2002, Bonneville, 2004). Bien que datée, une telle expression pourrait faire office de vérité intemporelle en matière de dynamique urbaine : « les villes, notamment dans la vieille Europe, tout autour de la Méditerranée, au cours de la durée, de leur longue durée, sont des villes qui n'ont cessé de se détruire et de se reconstruire sur elles-mêmes » (Burgel, 2001).

6 Si l'on met de côté les paramètres les plus matériels de la destruction, liés à l'usure des matériaux (constructions en bois inflammables, vieillissement des édifices etc.), aux considérations stratégiques (destructions liées aux guerres) ou économiques (destructions comme élimination d'un stock), les motifs de démolitions/reconstruction sont puissants.

7 Les normes et les usages évoluent (on parle volontiers d'innovations), qu'elles soient traduites en terme de besoins (confort, hygiène), de fonctionnalités (évolution des modes 
de production) ou de pratiques culturelles (bâtiments cultuels, lieux publics etc.). Dans l'esprit de la Charte d'Athènes, qui précisait « qu'en aucun cas le culte du pittoresque et de l'histoire ne doit primer sur la salubrité du logis » (Le Corbusier, 1957), les destructions opérées dans les quartiers de centre ville taudifiés (La Doutre à Angers, Le Vieux Mans, etc.) ont été justifiées par des considérations hygiénistes, liées notamment à la densité du bâti (accès à la lumière, absence d'aération). Quand on détruit une barre aujourd'hui dans un quartier d'habitat social, l'argumentaire porte notamment sur l'obsolescence des normes de construction fonctionnalistes des années 1960, que ce soit le caractère collectif du bâti ou la petite taille des appartements (de la cuisine notamment).

8 Les grandes phases de démolitions/reconstructions procèdent de logiques économiques et s'inscrivent dans des cycles bien décrits par J.-P. Lévy (1987) : profonds remaniements de la ville médiévale aux XVII ${ }^{\mathrm{e}}$ et XVIII ${ }^{\mathrm{e}}$ siècles, avec l'essor du capitalisme concurrentiel, et au XIX siècle, avec la Révolution industrielle (haussmannisation); recyclage d'espaces en déprise économique et sociale dans les péricentres et les banlieues des villes actuelles, dans un contexte d'affirmation d'une économie postindustrielle et d'un capitalisme financier mondialisés.

9 La destruction/reconstruction est également une opération économique en soit, qui suscite une activité importante (démolir, puis reconstruire), dans un secteur stratégique (le bâtiment) et qui peut créer les conditions d'une revalorisation foncière et immobilière. Même si l'on parle beaucoup de patrimoine aujourd'hui, une démolition/reconstruction apparait bien souvent plus rentable qu'une restauration avec remise aux normes : c'est ce qui s'est produit par exemple dans le cas de la caserne Desjardins à Angers, dont le principe de réutilisation, proposé par une étude, n'a pas été retenu par la mairie pour des raisons d'abord financières (Bergel et Veschambre, 2003).

Dans le même temps, la dimension symbolique est toujours présente à travers une démolition : «Les sociétés ne se sont-elles pas construites, d'abord en démolissant et en rebâtissant? » (Burgel, 2001). En effet, quelle meilleure manière pour un nouveau pouvoir de s'affirmer ou de marquer son temps, que de démolir et reconstruire par-dessus? Plus largement, la démolition, la table rase, exprime bien souvent la tentation de tirer un trait sur ce qui renvoie à des populations jugées indésirables ou à des souvenirs douloureux ou dévalorisants. C'est ainsi que l'on a annoncé du côté états-unien, la volonté de démolir la prison d'Abou Ghraïb à Bagdad, quelques temps seulement après qu'en soient sorties les révélations de tortures (Entous, 2004). Les conflits armés révèlent cette tentation de l'effacement de l'autre, non seulement physiquement mais également symboliquement, par la destruction architecturale et urbanistique, ce que les guerres en ex-Yougoslavie (Chaslin, 1997) ou en Palestine illustrent parfaitement: "par temps de guerre, de conquête coloniale, s'approprier ou altérer un territoire revient à le dépouiller non seulement de ses formes urbaines, de son architecture mais de sa mémoire collective » (Bulle, 2002).

11 Les changements de régime politique sont bien entendu propices à des destructions significatives d'une volonté d'effacement. Le processus de décolonisation s'est parfois accompagné de démolitions à forte charge symbolique: c'est ainsi que le palais du gouverneur japonais de Séoul a été « décapité » avant d'être détruit, après la défaite de la puissance occupante (Hsia, 2001). R. Robin (2003) a bien montré l'effacement méthodique des symboles de la RDA, à travers notamment les démolitions dans la capitale berlinoise, avec notamment le remodelage de ce haut lieu que représente l'Alexanderplatz. La démolition est très étroitement liée à la question de la mémoire, de l'identité collective et 
de la légitimité (Chivallon, 1999). Si cela paraît évident dans des contextes conflictuels, cette lecture en terme d'effacement se vérifie également dans la dynamique urbaine ordinaire, comme nous aurons l'occasion de le préciser un peu plus loin.

\section{Conservation : une idée occidentale qui tend à s'affirmer et à se diffuser dans la longue durée}

12 Même si l'histoire urbaine européenne est sans doute histoire de destructions avant que d'être histoire de pétrification, il est assez évident que dans le même temps, l'attachement à la matérialité, à la pierre, caractérise les sociétés occidentales. Le pouvoir s'incarne dans la pierre depuis les civilisations grecques et romaines, notamment dans le centre de la ville, en une sorte de fétichisation monumentale, le monument correspondant étymologiquement à « ce qui fait se souvenir ». Dans les sociétés asiatiques ou africaines, le rapport à la mémoire, à la transmission est beaucoup plus immatériel (Gravari-Barbas et Guichard-Anguis, 2003). De nombreux chercheurs ont montré l'opposition entre la conception occidentale du patrimoine d'une part, fondée essentiellement sur l'authenticité de la "substance", de la matière dans laquelle le patrimoine est construit, et les conceptions en vigueur en Extrême-Orient ou en Afrique d'autre part, où cette substance a finalement peu d'importance comparativement aux savoir-faire, aux gestes, à la culture etc. (Ouallet, 2000). Alors que l'Europe a hérité de milliers d'églises de l'époque romane, dont on va commencer à célébrer le millénaire, la société japonaise reconstruit périodiquement ses sanctuaires en privilégiant la forme, la symbolique plutôt que la matérialité.

13 Cette idée de conserver les héritages bâtis a cependant une histoire, qui est étroitement liée à celle des destructions. En France, c'est la Révolution et les destructions symboliques qui l'ont accompagnée, qui ont fait émerger un intérêt des élites pour la conservation des héritages de l'Ancien Régime, intérêt qui a commencé à s'institutionnaliser sous la monarchie de Juillet (Leniaud, 2002). Mais c'est depuis une bonne vingtaine d'années que la revendication patrimoniale s'est intensifiée dans les sociétés occidentales et s'est "démocratisée " pour englober des héritages moins «monumentaux» et plus récents, que l'on désigne bien souvent par l'appellation de "petit patrimoine ». Dans le même temps, depuis l'instauration des secteurs sauvegardés en 1962, la reconnaissance patrimoniale a pris une dimension spatiale évidente, en s'élargissant, au-delà des édifices ponctuels, à des ensembles urbains (Veschambre, 1998).

Ce mouvement est à mettre en relation avec les changements profonds dans les modes de production, induisant la désaffectation d'espaces agricoles (le bocage, les fermes), industriels (mines, usines sidérurgiques, textiles etc.), résidentiels (cités ouvrières, habitat populaire de centre ville etc.). Cette intensification de la revendication patrimoniale est à la mesure de l'accélération des évolutions technologiques, économiques et culturelles de ces cinquante dernières années. Ce qui devient patrimoine, c'est ce qui est désaffecté et qui tend à disparaître : «c'est la conscience de la disparition qui éveille l'intérêt patrimonial » (Carballo et Emelianoff, 2002).

Dans un tel contexte de profonde transformation des modes de production, la conservation ou non de tel ou tel héritage architectural, dans la culture occidentale pour le moins, revêt un enjeu mémoriel et identitaire de plus en plus affirmé. Avec de fortes inégalités dans la capacité à accéder au "conservatoire de l'espace » selon la position sociale (Verret, 1995). Car même si le champ patrimonial s'est considérablement élargi, 
les formes les plus élitistes (châteaux, maisons de maîtres, édifices publics, lieux de culte) demeurent prépondérantes parmi les édifices protégés par l'État, alors qu'à l'autre extrémité de la hiérarchie sociale, «la patrimonialisation s'arrête aux portes des quartiers d'habitat social» (Carballo et Emelianoff, 2002). Si la question de la patrimonialisation de l'habitat social des années 1950-1960 ne va effectivement pas de soi, (Veschambre, 2000a), ce désintérêt vaut également pour des formes plus anciennes d'habitat populaire, du type cités ouvrières (Duchêne, 2005).

Dans le même temps, ce succès de la patrimonialisation reste fortement lié à une logique de revalorisation, à la fois symbolique et économique, de pans entiers du tissu urbain. Comme l'écrit A. Bourdin (1992), « le patrimoine architectural est [...] ce qui donne valeur à un lieu ». Un travail collectif d'analyse lexicale, réalisé à partir d'un corpus de déclarations d'associations à caractère patrimonial et environnemental localisées dans les Pays de la Loire, nous avait d'ailleurs montré combien le mot patrimoine est étroitement associé à "valeur/valorisation» dans le discours associatif ${ }^{2}$ (Veschambre, 2000b). Certains auteurs ont d'ailleurs souligné que le succès du mot patrimoine au sens d'héritage culturel collectif, était pratiquement concomitant de celui de richesse individuelle transmissible (Andrieux, 1997). Le moteur de la patrimonialisation est bien souvent celui de la (re)valorisation d'espaces désaffectés et de leur transformation en ressource économique : «la valorisation du patrimoine, voire sa "marchandisation", est aujourd'hui un levier essentiel pour l'aménagement et le développement des territoires » (Chevallier, 2000).

17 La patrimonialisation apparaît bien au bout du compte comme une modalité, de plus en plus légitime, d'appropriation de l'espace. Comme le résume J. Chevalier (1999), le patrimoine renvoie à « l'appropriation de manière sélective, sur un mode symbolique, de la matérialité naturelle ou construite héritée ».

18 Ce décalage entre la rigidité relative du bâti urbain et les mutations sociales et économiques, plus ou moins rapides et amples selon les époques, s'est traduit depuis l'apparition des villes par un balancement entre destructions et conservation. Même si en Occident, la tendance semble être depuis deux siècles à la légitimation de la conservation, même si la période actuelle semble globalement moins "destructrice» que celle des années 1950-1960, il faut prendre du recul par rapport aux discours et aux affichages. Le recyclage par la démolition demeure le principal levier de transformation urbaine, notamment dans le cadre des vastes emprises désaffectées des espaces péricentraux. Alors que la centralité n'a cessé de se renforcer dans une logique de métropolisation, les intérêts économiques en jeu sont considérables et la tentation de la table rase est puissante, non seulement dans le contexte des villes du Sud ou des nouveaux mondes, mais également en Europe et en France. La logique du « développement durable », qui est censée orienter les politiques urbaines ${ }^{3}$, en France notamment, présente une ambiguïté qui laisse la porte ouverte à des interprétations très contradictoires : entre les logiques de densification et donc de démolition/reconstruction («reconstruire la ville sur la ville») et les logiques de conservation du tissu urbain, avec les incitations à intégrer plus fortement la connaissance et la protection du patrimoine dans les plans locaux d'urbanisme (PLU).

19 Nous allons revenir sur les enjeux liés au recyclage urbain, sous l'angle spécifique de l'appropriation ou de la réappropriation de l'espace. 


\section{Recyclage et appropriation de l'espace urbain}

20

Démolition et conservation constituent les deux faces d'un même processus de réinterprétation et de revalorisation permanente des héritages urbains. Nous les traiterons désormais de manière concomitante en remettant l'accent sur les enjeux d'appropriation de l'espace. Alors que les héritages urbains les plus anciens ont acquis dans la plupart des cas le statut de patrimoine, ce sont les constructions contemporaines ( $\mathrm{XIX}^{\mathrm{e}}-\mathrm{XX}^{\mathrm{e}}$ siècle) qui sont l'objet de l'essentiel du recyclage : nos exemples porteront pour la plupart sur ce type d'héritage.

\section{Obsolescence et recyclage : désaffectation et conflits d'appropriation}

Les changements d'usage, lorsqu'une activité économique ou une fonction sociale (habitat, pouvoir politique, expressions culturelles, cultuelles etc.) apparaissent obsolètes, sont généralement l'occasion d'une «redistribution des cartes", de conflits d'appropriation des espaces ainsi laissés vacants (Veschambre, 2000a). C'est précisément au moment de ces mutations économiques et sociales, dans une situation de vacance, de désaffectation de ces espaces, que se jouent la réaffirmation ou la remise en question des pouvoirs qui s'y exerçaient au préalable et dans le même temps le type de traitement de ces héritages, entre logique de la table rase et logique de la conservation. Cette désaffectation suscite l'intérêt de certains groupes, de certains acteurs (collectivités locales, fonctionnaires du ministère de la Culture, promoteurs, associations d'habitants, professionnels du tourisme etc.), qui se retrouvent ainsi en concurrence. C'est en effet l'occasion de s'approprier, ou de se réapproprier des espaces, afin d'en retirer des bénéfices symboliques, en terme d'image de marque, et/ou des bénéfices économiques liés à de nouvelles activités.

Le tour d'horizon réalisé dans les principales villes de l'Ouest nous a permis de repérer les conflits les plus significatifs de ces dernières années. Dans un processus où les communes (et de plus en plus les communautés d'agglomération) se retrouvent en première ligne dans les projets de «régénération » urbaine, c'est fondamentalement la capacité de ces collectivités locales à se réapproprier des espaces devenus stratégiques et centraux (île Sainte-Anne à Nantes, rives de la Maine à Angers, port à Caen, quartier de la gare au Mans etc.) et à y imprimer leur conception de l'urbanité, qui est mise en jeu. Dans ces différentes villes, les conflits liés au recyclage urbain ne sortent généralement pas des cercles du pouvoir et des spécialistes: ils se résument alors à un bras de fer entre des fonctionnaires de l'État (ministère de la Culture) chargés de faire valoir l'élargissement des conceptions du patrimoine et des collectivités territoriales, soucieuses de garder les mains libres, voire de conquérir une marge de manœuvre croissante.

C'est ce qui s'est produit à Angers en 2000, dans le cadre de la ZAC "Thiers-Boisnet » (fig. 1), au sein d'un quartier né de l'activité portuaire au milieu du xix ${ }^{e}$ siècle. Dans ce secteur artisanal et industriel, que la mairie voulait à l'origine entièrement démolir, le conflit s'est cristallisé sur une maison de chef d'entreprise, qualifiée "d'immeuble remarquable » dans l'étude d'impact (Veschambre, 2002b). La municipalité avait racheté cette maison pour la démolir et pour offrir au promoteur un îlot entièrement débarrassé de toute construction. S'appuyant sur l'étude d'impact, l'architecte des bâtiments de 
France $(\mathrm{ABF})$ a refusé le permis de démolir, ce qui a conduit le maire à déposer un recours auprès du préfet. L'affaire a été portée devant la Commission régionale du patrimoine et des sites (CRPS), qui a considéré que le projet d'ensemble de la ZAC n'avait pas été assez pensé et qui a également refusé la démolition. Au delà même des enjeux économiques, qui sont évidents dans cette opération d'intégration d'un ancien "bas quartier portuaire " dans un centre ville élargi, ce sont deux conceptions de la ville qui se sont affrontées : l'ABF considérant que les héritages du XIX ${ }^{e}$ siècle devaient structurer la rénovation du quartier, tandis que la mairie, par l'intermédiaire de son architecte, affirmait que les formes urbaines contemporaines devaient imposer une nouvelle structuration du tissu urbain, dans une dénégation de l'intérêt des héritages, notamment industriels.

Le préfet de Région a finalement cassé la décision de la CRPS et accordé le permis de démolir, ce qui est rarissime. Cela a donc été l'occasion pour la mairie d'Angers d'affirmer sa maitrise de l'espace communal et d'y marquer son empreinte, en s'opposant victorieusement à l'imposition des normes venues « d'en haut ».

Figure 1 : Les principales démolitions dans le centre-ville d'Angers (1960-2005)

The most important demolitions in the centre of Angers (1960-2005)

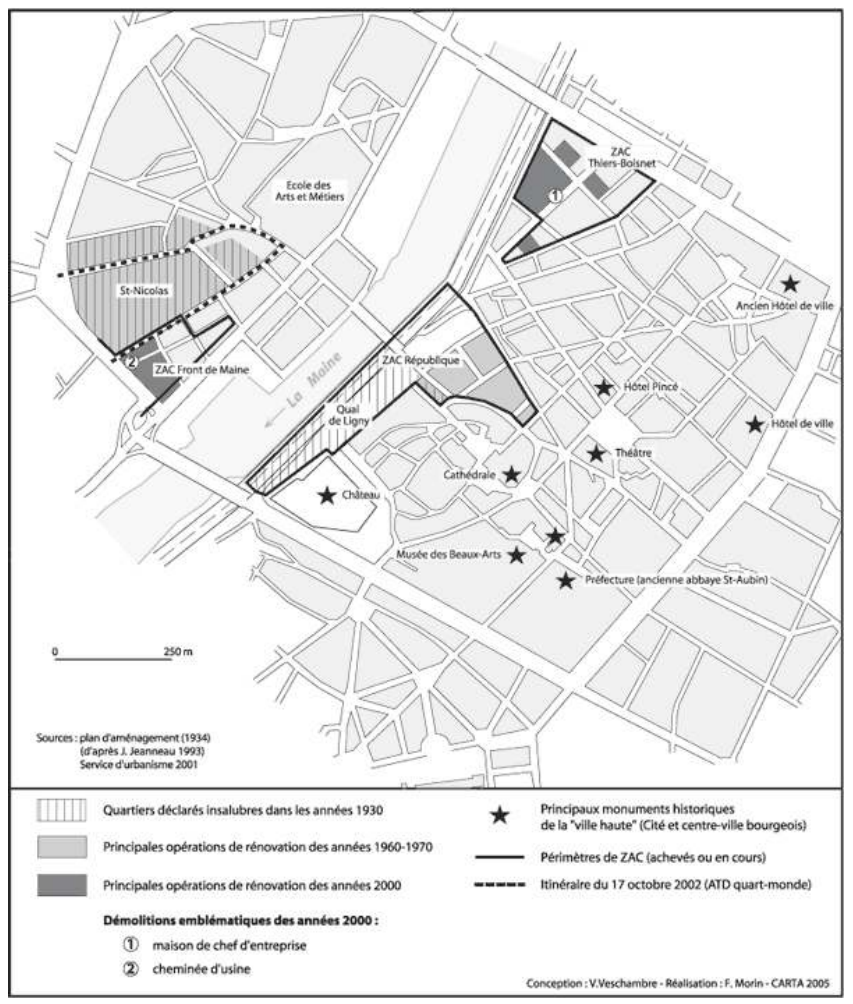

Dans ce type de bras de fer, c'est l'inverse qui s'est produit au Mans, à propos de deux édifices tout à fait emblématiques. L'asile d'aliénés Etoc-Demazy a fait l'objet de discussions pendant une dizaine d'années, avant que son classement soit imposé en 2001, compte tenu de son importance dans l'histoire de l'architecture hospitalière. La mairie a tout fait pour éviter un tel classement et les services de l'urbanisme continuent de déplorer la présence d'un périmètre monument historique dans le cadre d'un quartier de gare promis à de profondes transformations dans les années futures. Le même processus est en train de se produire avec la gare routière, construite en 1935, qui figure sur la liste 
label xxe siècle dressée par la DRAC en 2004, pour sa façade (fig. 2) et sa coupole de béton armé et de verre.

Figure 2 : La gare routière au Mans (label $x x^{e}$ siècle) (cl. V. Veschambre, nov. 2004) The bus station in Le Mans ( $X X^{\text {th }}$ century label)

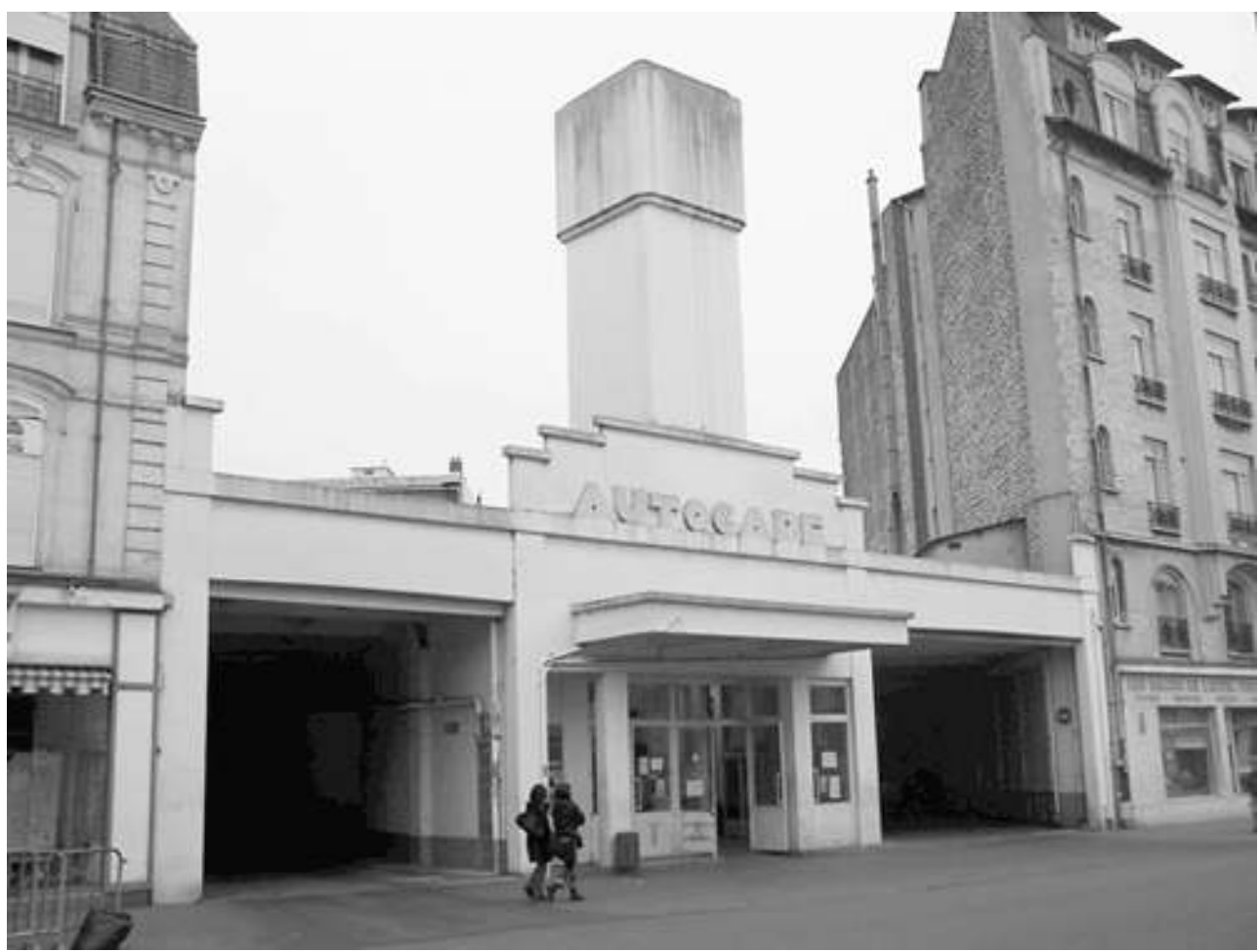

Alors que la compagnie de transports privée qui l'exploite va bientôt abandonner les lieux, la mairie avoue son incapacité à proposer un nouvel usage et redoute l'installation d'une friche dans un secteur hautement spéculatif, toujours en rapport avec le tracé du tramway. Suite à cette reconnaissance patrimoniale, la mairie considère être dépossédée de son pouvoir de façonner la ville.

Dans les villes moyennes que nous avons étudiées (Angers, Le Mans, Caen) les conflits liés au recyclage urbain qui engagent les habitants sont assez peu nombreux. Ce n'est sans doute pas un hasard si deux des conflits les plus intéressants concernent des édifices religieux. À Angers, la seule mobilisation significative de ces quinze dernières années s'est produite dans le cadre de la ZAC Saint-Lazare, du nom d'un faubourg situé dans le prolongement du quartier de la Doutre. L'ancienne léproserie Saint-Lazare, chapelle du $\mathrm{XII}^{\mathrm{e}}$ siècle, a été redécouverte derrière la devanture d'un commerce racheté par la mairie et voué à la démolition. L'association « Renaissance de la chapelle Saint-Lazare » a réussi à faire plier la mairie, qui voyait là un obstacle à sa refonte du quartier. À travers cet édifice emblématique, qui avait donné son nom au quartier, les habitants ont trouvé un levier pour s'immiscer dans le programme de rénovation et se réapproprier une certaine capacité d'intervention.

$\mathrm{Au}$ Mans, c'est une chapelle du xix ${ }^{e}$ siècle, Saint Joseph, propriété de l'évêché qui ne souhaitait plus l'entretenir, qui a fait l'objet en 2004 d'un conflit public. Après avoir hésité, la mairie a finalement accordé le permis de démolir, ce qui a été vivement contesté par des habitants du quartier, qui ont réussi pour l'instant à faire revenir l'évêché sur sa décision. Comme dans tous les conflits de ce type, la mobilisation est 
impulsée par des individus qui ont légitimité pour le faire, qui disposent d'un certain nombre de capitaux et se positionnent ainsi sur la scène publique locale, en investissant symboliquement des lieux visibles (Veschambre, 2000a) : en l'occurrence, c'est l'ancien directeur du Conseil d'Architecture, d'Urbanisme et de l'Environnement (CAUE) de la Sarthe, par ailleurs homme politique affilié à l'UMP, qui a impulsé la mobilisation.

Dans notre échantillon de villes, Nantes fait exception par l'ampleur et le nombre des mobilisations associatives qui ont émergé dans le cadre des politiques de renouvellement urbain. L'existence d'un collectif d'associations autour du patrimoine industriel apparait tout à fait exceptionnelle dans le contexte français (André et Kerouanton, 2003) et a pesé fortement sur la vie politique locale et sur l'évolution des conceptions et pratiques de la municipalité actuelle en matière de réaménagement urbain. Cette intervention a porté notamment sur un ancien pôle industrialo-portuaire situé au sud du centre ville, aujourd'hui rebaptisée île de Nantes et progressivement transformé en espace tertiaire de commandement (Peyon, 2000). C'est ainsi que l'ancienne gare de l'État ou des éléments des chantiers navals Dubigeon (fermés en 1987), ont été sauvés de la démolition par des manifestations impulsées par ce tissu associatif, issu à la fois des milieux ouvriers et syndicaux, universitaires et patronaux. Alors que la logique financière était prioritaire dans le projet de renouvellement urbain de la fin des années 1980, centré sur une Cité internationale des affaires ${ }^{4}$, la nouvelle équipe municipale a dû laisser place à une réappropriation symbolique partielle de ces friches industrielles, par ceux qui se sentent dépositaires de la mémoire industrielle et ouvrière.

Ces différents exemples (chantiers navals, gare routière, hangars et maison d'industriel, lieu de culte etc.) nous rappellent combien le processus de recyclage de l'espace urbain est fondamentalement lié à la désaffectation consécutive à des changements de mode de production et d'organisation sociale. Ce qui suscite des intérêts contradictoires en matière de contrôle et d'appropriation des espaces ainsi rendus disponibles.

\section{Recyclage urbain et légitimation d'une appropriation de l'espace : des processus de marquage}

31 Nous reviendrons pour finir sur les modalités selon lesquelles une appropriation de l'espace est signifiée, dans le registre symbolique, à l'occasion d'un recyclage du tissu urbain.

Une telle manifestation symbolique d'une appropriation passe par la production d'un marquage, c'est-à-dire par une transformation matérielle de l'espace, plus ou moins durable, à des fins signifiantes (Veschambre, 2004). Pour la commodité du raisonnement et au risque de décomposer des mécanismes qui sont souvent imbriqués, nous distinguerons trois grands types de marquage sur le plan urbanistique et architectural.

\section{Produire les marques d'une appropriation}

La forme privilégiée d'affirmation d'un pouvoir sur un espace, c'est l'acte urbanistique et architectural. Comme ce sont des espaces stratégiques qui sont visés en priorité, cela suppose des interventions sur des tissus urbains denses et donc le plus souvent des démolitions. 

Palais de Justice. «Signe manifeste d'autorité » selon le président du tribunal de grande instance, le Palais de justice de Nantes, construit par Jean Nouvel et inauguré en 2000 (fig. 3), illustre cette volonté de l'institution judiciaire de s'affirmer symboliquement par la monumentalité de ses réalisations (Lamarre, 2004). Les emplacements choisis manifestent la volonté du ministère de la Justice de maintenir ses juridictions au centre des villes, près des autres lieux de pouvoir. Au Mans, les volumes cubiques de la Cité judiciaire sont tournés vers le chevet de la cathédrale. À Caen, le ministère a décliné des offres foncières périphériques pour s'inscrire dans la ZAC Gardin, entre l'hôtel de ville (Abbaye aux hommes) et la préfecture. À Nantes, le Palais de Justice renforce le nouveau pôle de centralité que constitue désormais l'île de Nantes.

Figure 3 : Le Palais de justice de Nantes construit par Jean Nouvel sur lîle de Nantes (cl. V. Veschambre, mars 2005)

The law courts built buy Jean Nouvel on Nantes Island

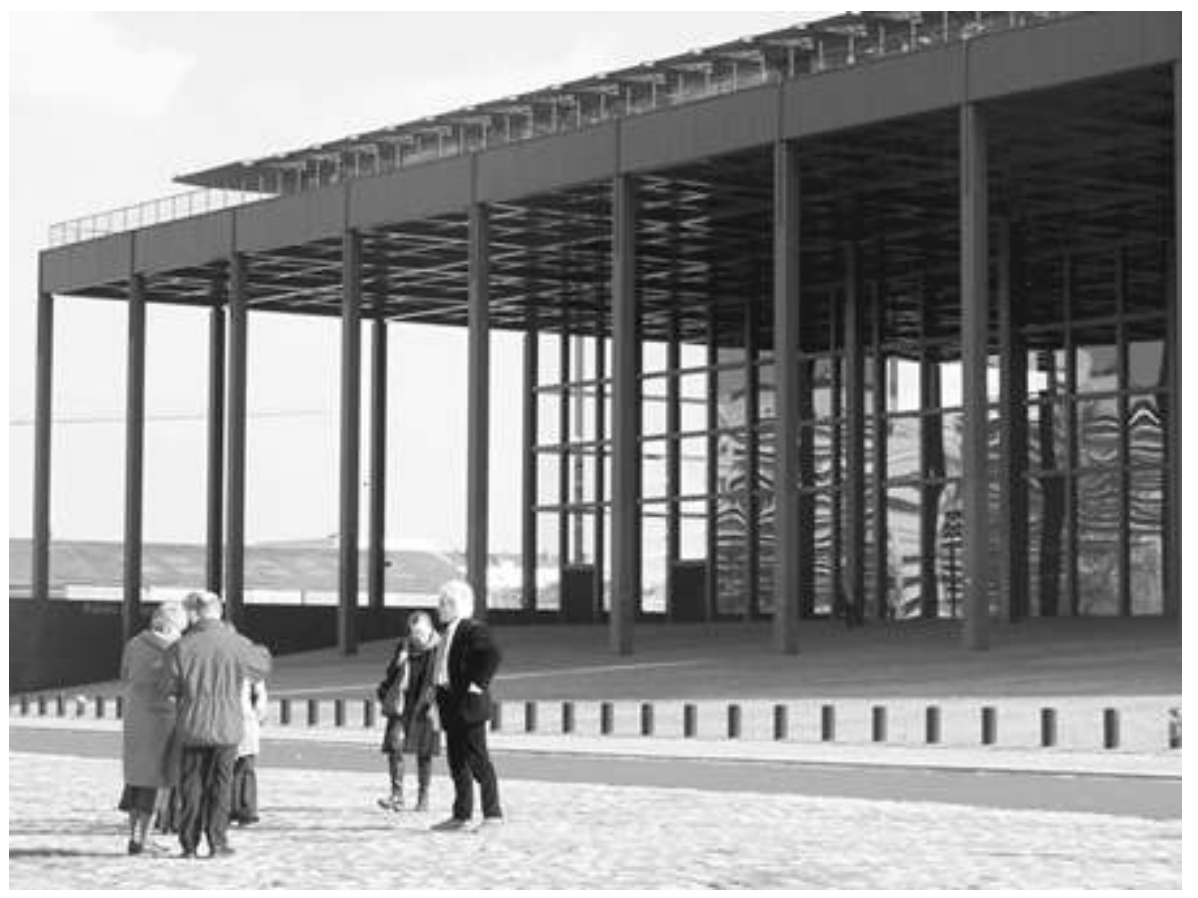

Dans un système politique où le pouvoir municipal est très personnifié, les maires ont le souci de marquer la ville de leur empreinte, que ce soit indirectement, par le biais d'ouvrages de prestige impulsés de l'extérieur (édifices d'État, constructions privées etc.) ou à travers des commandes directes. À Caen, l'ancien maire Jean-Marie Girault a été à l'origine d'un nouveau stade et d'un Zénith, qui figurent depuis en bonne place dans la communication de la mairie. Parmi les nombreux chantiers de l'ancien maire d'Angers, Jean Monnier, signalons le quartier du Front de Maine, dessiné par Claude Vasconi, face au château ; tandis que le nom du maire actuel, Jean-Claude Antonini, restera associé au futur théâtre Le Quai, conçu par l'équipe parisienne Architecture Studio, dans le cadre de la même ZAC du Front de Maine (fig. 1).

\section{Le marquage patrimonial : la logique de la restauration}

La production de marques passe également par des restaurations de prestige. Alors qu'à Angers les maires des années 1960 aux années 1980 se sont signalés par d'importantes 
démolitions dans le centre ville (les halles et le quartier République, les abattoirs, les Bains douches etc.), le maire actuel mise beaucoup sur la restauration du musée des Beaux-Arts (Logis Barrault, d'époque Renaissance) pour communiquer autour du « développement durable » et laisser sa marque (fig. 1).

La patrimonialisation produit elle aussi une forme de marquage, constituant le support d'une appropriation de l'espace (Veschambre, 2000a). L'intervention matérielle qui suit la reconnaissance d'un héritage architectural (réhabilitation, restauration des façades notamment) modifie l'édifice et constitue une signature: celle des autorités qui l'ont financée, mais aussi celle des groupes sociaux qui ont promu la reconnaissance de ce patrimoine. À Nantes, la gare de l'État et les bâtiments de direction des chantiers Dubigeon, occupés par des syndicats et des associations qui s'intéressent à la mémoire ouvrière, en constituent un très bon exemple. Dans le même secteur, les anciennes usines LU, rebaptisées Lieu Unique et réhabilitées en tant qu'espace culturel, constituent la vitrine d'une politique municipale, sur le plan culturel, patrimonial et urbanistique.

\section{La démolition : un processus d'effacement mémoriel}

Produire une marque dans l'espace, à travers la construction ou la restauration d'édifices, a pour objectif plus ou moins conscient de laisser une trace et de faire mémoire. Et inversement, il y a bien évidemment des logiques d'effacement de traces dans la démolition, suivie ou non par la production de nouvelles marques. Il y a dans le recyclage de l'espace urbain des enjeux évidents de revendication mais aussi de négation mémorielle, liés à l'invocation ou à l'effacement des traces. Ceci dans un contexte d'affirmation généralisée du registre mémoriel, comme vecteur de revendication, de visibilité et de légitimité des groupes sociaux.

La démolition d'îlots insalubres dans les centres-villes anciens, qui a fortement marqué les politiques urbaines $d u x^{e}$ siècle, jusque dans les années 1970, peut être interprétée à la fois comme une forme d'éviction des catégories populaires (Levy-Vroëlant, 1999), mais aussi comme l'effacement symbolique de ces populations dans la mémoire urbaine. C'est ce que nous avons pu observer dans le contexte angevin, dans le cadre d'un travail mené avec le mouvement ATD quart-monde (Veschambre, 2002a). Lorsqu'en 2001 le mouvement est parti en quête de lieux de mémoire pour célébrer la journée internationale du refus de la misère, il s'est orienté vers deux quartiers associés historiquement à la pauvreté : le quai Ligny, qui était situé entre le château et la Maine, et le quartier Saint-Nicolas dans la Doutre, deux secteurs identifiés comme insalubres dès les années 1930 (fig. 1). Mais ces deux quartiers ont fait l'objet de rénovations urbaines radicales à la fin des années 1960 et au début des années 1970. Rien ne subsistant du quai Ligny, à l'emplacement duquel passe la voie rapide, ATD s'est tourné vers la Doutre. Nous avons identifié quelques traces de l'activité industrielle et artisanale aux abords du quartier reconstruit de Saint-Nicolas, qui ont servi de point de repère à l'évocation d'un quartier anciennement populaire, aujourd'hui complètement assimilé au centre ville par le prix de l'immobilier et le type de population. Ces traces, intégrées au parcours du 17 octobre 2001, ont été démolies au printemps 2004, pour laisser place au chantier du théâtre Le Quai. Parmi ces traces figurait la dernière cheminée d'usine du centre ville et l'une des toutes dernières de la commune (fig. 4). 
Figure 4 : Démolition de la dernière cheminée du centre ville (ancienne usine Laboulais et ancien théâtre Beaurepaire) en juin 2004. À l'arrière plan : immeubles de la rénovation de Saint-Nicolas (cl. V. Veschambre, 2004)

The demolition of the last factory chimney in the center of Angers

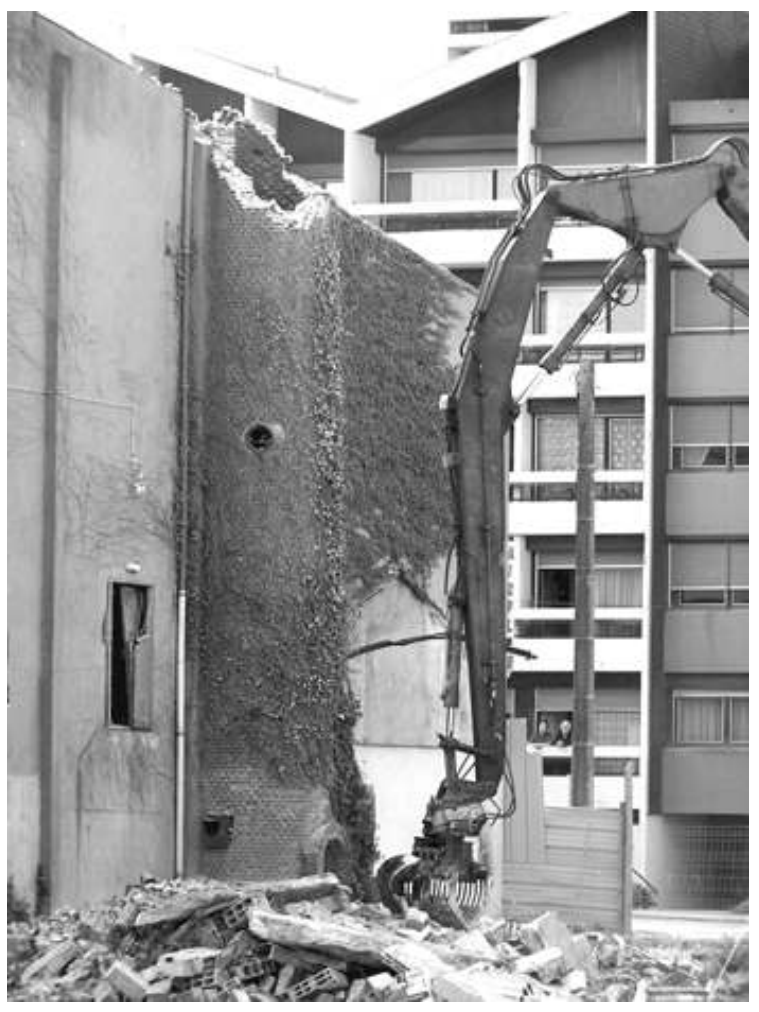

Alors qu'à Nantes est engagé depuis 20 ans un processus de reconnaissance et de mise en valeur des principaux témoins de l'histoire industrielle, les choix urbanistiques effectués à Angers ont fait pratiquement disparaitre ces témoins du paysage urbain ; c'est ainsi qu' « on a oublié qu'Angers était une ville industrielle ${ }^{5} »$, dominée par le textile. Si l'on se réfère aux 16 usines qui figurent sur le Nouveau plan d'Angers monumental, industriel et commercial de 1905, ne subsistent aujourd'hui que les bâtiments d'une ancienne manufacture de chaussures ${ }^{6}$. La démolition dans les années 1960 des immenses usines Bessonneau, qui ont employé jusqu'à 10000 ouvriers dans la filature et la corderie, fausse aujourd'hui la lecture de l'histoire urbaine. La référence au passé industriel semble reléguée à la périphérie de l'agglomération, dans les anciennes banlieues ardoisières de Saint-Barthélemy et surtout Trélazé, où la communauté d'agglomération vient d'acquérir 200 hectares de friches.

Cette situation se retrouve au Mans, où les témoins du passé industriel, comme les usines Bollée, ont été largement démolis dans la ville-centre de l'agglomération.

Plus que tout autre type d'héritages architecturaux, les anciennes unités de production sont exposées à la démolition. Placés sous le signe de la fonctionnalité, constitués bien souvent de vastes édifices associés à des activités qui étaient généralement pénibles et génératrices de nuisances, ces héritages sont plus difficiles à réinvestir, tant du point de vue matériel que symbolique. Sans parler de la rentabilité d'une opération de "requalification", il y a une certaine facilité à effacer ce qui renvoie à une mémoire douloureuse (la désindustrialisation, les conditions de travail etc.) et socialement peu valorisante (le monde ouvrier). 
Cette question du marquage symbolique de l'espace qui est opérée à la faveur du recyclage urbain peut constituer une grille de lecture des rapports sociaux. Alors que les groupes sociaux possédants et dominants peuvent matérialiser leur existence et leurs positions sociales dans les espaces les plus prestigieux (constructions, restaurations etc.), les groupes les plus démunis et dominés n'ont pas la maîtrise de leur espace résidentiel, qu'il soit réhabilité et réapproprié (centres anciens) ou qu'il soit démoli («îlots insalubres ", quartiers d'habitat social [Moncomble, 2002]). Ces derniers ne peuvent laisser au bout du compte que peu de traces patrimonialisables.

\section{Conclusion}

44 À travers ces quelques exemples de démolition et de patrimonialisation, nous avons essayé de montrer que le renouvellement urbain était non seulement l'occasion d'une « redistribution des cartes » du point de vue juridique et économique, mais qu'il pouvait être également interprété sur le plan de la (ré)appropriation symbolique de l'espace. Renforcement d'un pouvoir municipal, maintien d'une présence de l'État, affirmation d'associations de quartier et de leaders d'opinion, promotion de la mémoire syndicale et ouvrière etc.: les nouvelles constructions ou les restaurations constituent le vecteur privilégié d'affirmation des différentes formes de pouvoir et des groupes sociaux localisés qui y sont associés. Ces processus de marquage de l'espace urbain expriment toute l'importance du travail idéologique de légitimation des groupes sociaux dans la dimension spatiale. Au-delà de l'expression d'une appropriation, c'est en effet la visibilité, l'existence sociale de ces groupes qui est en jeu à travers des formes de marquage durables.

Cette capacité à s'inscrire durablement dans la pierre et dans la ville est extrêmement inégale. Si l'on excepte l'exemple nantais, où la mémoire ouvrière a acquis droit de cité par la mobilisation et l'inscription dans le jeu politique local, les autres villes étudiées se caractérisent par la négation de leur passé industriel et dans le même temps, par une occultation de cette mémoire ouvrière.

\section{BIBLIOGRAPHIE}

ANDRÉ (B.), KEROUANTON (J.-L.), 2003. - « Entretien avec Jean-Marc Ayrault, maire de Nantes », L'Archéologie industrielle en France, $\mathrm{n}^{\circ}$ 41, p. 78-83.

ANDRIEUX (J.-Y.), 1997. - Patrimoine et histoire, Paris, Belin, 283 p.

BERGEL (P.), 2002. - Quand la ville se refait. Renouvellement urbain et grandes emprises foncières. Études de cas en France métropolitaine et dans deux agglomérations de province: Caen et Angers, thèse de doctorat, soutenue le 17 décembre 2002, Université de Caen.

Bergel (P.), Veschambre (V.), 2003. - «L'Armée dans la ville : vers un renouvellement des rapports entre l'urbain et le militaire (le cas des agglomérations d'Angers et de Saumur) », dans LEROUX 
(P.) (dir.), Présence et représentation de l'institution militaire dans la ville, Les Documents $d u C 2 S D, n^{\circ} 54$, mars 2003, p. 13-97.

BONNEVILLE (M.), 2004. - « Les ambiguïtés du renouvellement urbain en France », Les Annales de la recherche urbaine, $\mathrm{n}^{\circ}$ 97, décembre 2004, p. 7-16.

Bourdin (A.), 1992. - « Patrimoine et demande sociale », dans NeYRET (R.) (dir.), Le patrimoine atout du développement, Presses universitaires de Lyon, $153 \mathrm{p}$.

BULLE (S.) 2002. - « Les villes palestiniennes entre passé colonial et avenir incertain », Les Annales de la recherche urbaine, $\mathrm{n}^{\circ}$ 91, p. 83-91.

Burgel (G.), 2001. - « Mémoire de ville et recomposition urbaine », dans LOYER (F.) (dir.), Ville d'hier, ville d'aujourd'hui en Europe: Actes des Entretiens du patrimoine, Paris, Éditions du Patrimoine/ Fayard, p. 95-104.

CARBAllo (C.), EMELIANOFf (C.), 2002. - « La liquidation du patrimoine, ou la rentabilité du temps qui passe ", Les Annales de la recherche urbaine, n 92, p. 49-58.

CHASLIN (F.), 1997. - Une haine monumentale, Paris, Descartes et $\mathrm{C}^{\mathrm{ie}}, 107 \mathrm{p}$.

CheVAlier (J.), FrÉMONT (A.), HÉRIN (R.), RenARD (J.), 1984. - Géographie sociale, Paris, Masson, 381 p. CheVAlIER (J.), 1999. - La médiation spatiale : les mots pour faire, les mots pour dire, ESO : travaux et documents, $\mathrm{n}^{\circ} 10$, mars 1999, p. 73-78.

Chevallier (D.), 2000. - « Avant propos », Campagnes de tous nos désirs, Paris, Éditions de la Maison des sciences de l'homme, coll. « Ethnologie de la France », Cahier 16, p. IX-XIV.

Chivallon (C.), 1999. - «Bristol et la mémoire de l'esclavage ", Annales de la recherche urbaine, $\mathrm{n}^{\circ}$ 85 , p. $100-110$.

DUCHÊNE (F.), 2005. - « Les anciennes cités ouvrières : entre patrimonialisation et normalisation », dans Gravari-barbas (M.) (dir.), Habiter le patrimoine. Enjeux, approches, vévus, Rennes, PUR, p. 517-526.

Dynamiques uRbaines, 1991. - Géographie sociale, Caen, Centre de publications de l'Université de Caen, $\mathrm{n}^{\circ} 11,455 \mathrm{p}$.

EnTous (A.), 2004. - « Bush tente de rassurer les Américains sur sa stratégie en Irak », Libération, 25 mai 2004.

GRAVARI-BARBAS (M.), GUICHARD-ANGUIS (S.), 2003. - Regards croisés sur le patrimoine dans le monde, Paris, Presses de l'Université de Paris-Sorbonne, 958 p.

HSIA (C.), 2001. - « Taipei, Séoul : culture urbaine et transformations », Communication orale au colloque Hanoï au miroir des métropoles asiatiques, IFA, Paris, 13 septembre 2001.

LAMARRE (F.), 2004. - Le Palais de justice de Nantes, [http://www.archimetal.com].

LE CORBUSIER, 1957. - La Charte d'Athènes, Paris, Minuit, 188 p.

LENIAUd (J.-M.), 2002. - Les archipels du passé, Paris, Fayard, 361 p.

LEVY (J.-P.), 1987.- Réflexions sur l'évolution contemporaine des centres-villes, BAGF, nº 4, p. 307-316.

LEVY-VRoÉLANT (C.), 1999. - « Le diagnostic d'insalubrité et ses conséquences sur la ville, Paris 1984-1960», Population, INED, p. 707-727. 
Moncomble (F.), 2002. - « Démolir la cité ; une ultime violence ? ", Les Annales de la recherche urbaine, $\mathrm{n}^{\circ} 92$ p. 41-48.

OUALLET (A.), 2000. - « Émergence patrimoniale et conflits en Afrique subsaharienne : l'exemple du Mali », Norois, n 185, p. 23-40.

PEYON (J.-P.), 2000. - « Patrimoine et aménagement urbain à Nantes : des relations conflictuelles permanentes ", Norois, $\mathrm{n}^{\circ} 185$, p. 113-124.

RoBIN (R.), 2003. - La mémoire saturée, Stock, Paris, 525 p.

VERRET (M.), 1995. - Chevilles ouvrières, Paris, Les Éditions de l'Atelier/Éditions ouvrières, coll. «Mouvement social », $254 \mathrm{p}$.

VesChAMBRe (V.), 1998. - « Le patrimoine : de la pierre au paysage », dans FouRniER (J.-M.), RAoulX (B.) (dir.), Environnement, aménagement, société en Basse-Normandie, Les documents de la Maison de la Recherche en Sciences Humaines de Caen, $\mathrm{n}^{\circ}$ 6, p. 55-76.

-, 2000a. - « Patrimonialisation et enjeux politiques : les édifices Le Corbusier à Firminy, in Patrimoine et environnement : les territoires du conflit », Norois, $\mathrm{n}^{\circ}$ 185, p. 125-138.

-, 2000b. - «Le paysage dans le discours associatif : une présence discrète ", actes du colloque Action paysagère et acteurs territoriaux, Poitiers, GESTE, décembre 2000, p. 39-48.

-, 2002a. - « Une mémoire urbaine socialement sélective, réflexions à travers l'exemple

d'Angers ", Les Annales de la recherche urbaine, $\mathrm{n}^{\circ}$ 92, p. 65-74.

-, 2002b. - « Une conception de l'urbanité destructrice des héritages industriels : la ZAC ThiersBoisnet à Angers ", ESO : travaux et documents, n 18, décembre 2002, p. 45-51.

-, 2004. - « Appropriation et marquage symbolique de l'espace : quelques éléments de réflexion ", ESO : travaux et documents de l'UMR 6590, n² 21, mars 2004, p. 73-77.

\section{NOTES}

1. Ce travail est réalisé dans le cadre du groupe «politiques patrimoniales » de l'UMR CNRS 6590 (ESO).

2. Alors que dans cette analyse, le terme « environnement " se situe dans un tout autre registre, celui de la « défense ", de la " protection ».

3. Dans le cadre notamment de la loi Solidarité et renouvellement urbain (SRU) pour ce qui concerne la France (13 décembre 2000).

4. C'étaient à l'époque la CCI (chambre de commerce et d'industrie) et le Port autonome qui donnaient l'impulsion, en lien avec un financier luxembourgeois (Peyon, 2000).

5. Citation d'un entretien réalisé avec Jean-Louis Kerouanton, responsable du patrimoine industriel et $\mathrm{xx}^{\mathrm{e}}$ siècle à la DRAC des Pays de la Loire. Il ne s'agit pas de placer Angers sur le même plan que le pôle industriel de l'estuaire, mais de rappeler que l'industrie textile a profondément marqué la ville, de la fin du XIX siècle aux années 1950.

6. Où sont logés pour l'instant les services de l'inventaire du patrimoine municipal. Il faudrait ajouter la présence d'un bâtiment des anciennes filatures du Clon, dans le quartier Lafayette. 


\section{RÉSUMÉS}

Nous proposons dans ce texte d'appréhender le processus de «renouvellement urbain» (démolitions, reconstructions, restaurations, réutilisations) en terme de (ré)appropriation symbolique de l'espace. Expression du pouvoir, la ville est en effet le théâtre d'une compétition permanente entre groupes sociaux, pour l'accès aux espaces les plus valorisés, non seulement sur le plan des avantages économiques, mais également sur le plan des ressources symboliques. Après avoir explicité le sens des démolitions dans cette logique de reconstruction de la ville sur elle-même et rappelé succinctement l'émergence et l'affirmation d'une sensibilité occidentale à la conservation des héritages architecturaux, au-delà de leur désaffectation, nous envisagerons conjointement ces deux faces d'un même processus de recyclage et de réappropriation de l'espace urbain. À travers quelques exemples choisis à Angers, Le Mans, Nantes et Caen, nous passerons en revue les principaux types d'intervention sur le bâti, en les interprétant comme des formes de marquage de l'espace, et par là même, de revendication d'une appropriation de cet espace. Nous mettrons en évidence des différences de perception et de mise en valeur du patrimoine, notamment du point de vue des héritages urbains les plus récents, la patrimonialisation représentant aujourd'hui un vecteur privilégié d'appropriation symbolique de l'espace.

This paper is aimed at analysing the process of urban renewal - destruction, reconstruction, restauration, re-utilisation - in terms of symbolic (re)appropriation of the space. As an expression of power, the city is the theatre of permanent competition between social groups for access to the best valued spaces not only due to their economic resources but also their symbolic resources.

Within this logic of permanent reconstruction, we attempt to give an explanation of the sense of destruction and briefly recall the emergence and affirmation of a Western sensitivity to the conservation of architectural heritage beyond its abandonment. We examine the two sides of the same process of recycling and reappropriation of urban space considering the examples of Angers, Le Mans, Nantes and Caen and we review the main steps of the reappropriation, providing evidence of the differences in perception and the enhancement of patrimony from one town to another, notably from the point of view of the most recent urban heritages.

\section{INDEX}

Index géographique : Angers, Caen, France, France de l'Ouest, Le Mans, Nantes Mots-clés : appropriation de l'espace, démolition, patrimoine industriel, patrimonialisation, ville

Keywords : appropriation of space, city, destruction, heritage, industrial heritage 


\section{AUTEUR}

\section{VINCENT VESCHAMBRE}

CARTA (Centre angevin de recherche sur les territoires et l'aménagement) - Université d'Angers, ESO, UMR 6590 - CNRS, vincent.veschambre@univ-angers.fr 